\title{
De la ciudad indígena Salduie-Salduvia a la colonia romana Caesar Augusta
}

\author{
Francisco Pina Polo \\ Universidad de Zaragoza \\ franpina@unizar.es
}

\section{RESUMEN}

Sobre la base de la ausencia total de menciones de Salduie/Salduvia en las fuentes literarias (con la excepción de Plinio), su aparición en inscripciones del siglo I (Bronce de Ascoli y tabula Contrebiensis) y la acuñación de monedas en torno al año 100 a.C., este artículo plantea la hipótesis de que Salduie/ Salduvia fuera fundada como ciudad indígena a finales del siglo II a.C., tal vez por sinecismo a partir de las poblaciones del entorno.

Palabras clave: Salduie/Salduvia. Caesar Augusta. Romanización. Fundación de ciudades.

\section{From the Indigenous Town of Salduie/Salduvia to the Roman Colony of Caesar Augusta}

\begin{abstract}
This paper suggests that the indigenous town of Salduie/Salduvia could be founded at the end of the second century BC, maybe by grouping populations of the surrounding area. This hypothesis is based on the total absence of Salduie/Salduvia in the ancient sources (with the exception of a text in Pliny), its mention in Latin inscriptions of the first century BC (Ascoli Bronze and tabula Contrebiensis), as well as the coinage of coins in the town around $100 \mathrm{BC}$.
\end{abstract}

Key Words: Salduie/Salduvia. Caesar Augusta. Romanisation. Foundation of towns. 
De acuerdo con Plinio, la colonia romana de Caesar Augusta fue fundada junto al río Ebro, en el solar de lo que antes era la ciudad de Salduvia, perteneciente al pueblo indígena de los sedetanos. ${ }^{1}$ Plinio no proporciona ninguna otra información sobre esa población. En realidad, es la colonia fundada por el emperador César Augusto la que realmente interesa al autor latino, siendo la mención de la ciudad indígena sobre todo un signo de su erudición. Durante décadas se discutió sobre la veracidad del texto de Plinio, hoy plenamente confirmado, puesto que han aparecido suficientes restos arqueológicos que demuestran que efectivamente existió en el mismo lugar una ciudad antes de la fundación de la colonia romana. ${ }^{2}$ Los hallazgos excavados pertenecen fundamentalmente a viviendas dotadas de elementos arquitectónicos y decorativos que indican un alto grado de desarrollo urbanístico, así como semejanzas con construcciones itálicas de la misma época. Desde un punto de vista cronológico, los restos hallados corresponden al siglo I a.C. De los hallazgos realizados hasta el momento difícilmente pueden extraerse conclusiones ciertas sobre el tamaño y la importancia de la ciudad indígena, pero el hecho de que todos ellos se encuentren situados en la zona nororiental de la antigua Zaragoza, en los barrios actuales de La Seo y La Magdalena, ha llevado a suponer que la ciudad indígena pudo estar situada exclusivamente en esa zona próxima a la desembocadura del Huerva en el río Ebro, una pequeña porción de lo que más tarde sería la colonia Caesar Augusta. ${ }^{3}$

La existencia de esa población indígena ha sido confirmada por los datos numismáticos. La ciudad a la que los romanos llamaban Salduvia acuñó monedas con el nombre de Salduie, que era la denominación con la que la conocían sus habitantes indígenas. ${ }^{4}$ Conocemos una única serie de monedas fragmentarias en bronce. ${ }^{5}$ Sin embargo, significativamente Salduie no acuñó en plata como sí lo hicieron otras cecas más importantes. Las acuñaciones conocidas fueron exclusivamente realizadas muy al final del siglo II y al comienzo del I a.C. Por otra parte, la difusión de las monedas de Salduie fue escasa, en cualquier caso mucho menor que la de otras cecas de su entorno, como Sekia (probablemente Ejea), Bolskan (Huesca), Turiasu (Tarazona), Sekaiza (Mara) y Kelse (la antecesora de la colonia Celsa en Velilla de Ebro).

Sorprende que, con la excepción de la ya citada mención de Plinio, la ciudad de Salduie-Salduvia no aparece nunca en las fuentes antiguas que han llegado hasta nosotros. Ciertamente, la obra de Livio se ha perdido a partir del año 167 a.C., momento desde el cual sólo se conocen resúmenes posteriores de sus libros. Esto podría ser una explicación para su silencio sobre Salduie, pero es un argumento débil e insuficiente, puesto que es precisamente en el primer cuarto del siglo II a.C. cuando el valle medio del Ebro adquiere un mayor protagonismo en la conquista de Hispania en el momento en el que Roma comenzó a chocar con los celtíberos. Por su parte, Apiano es indudablemente una fuente de información mucho menos detallada que Tito Livio, pero, a cambio, es el único relato continuado de la conquista de Hispania que ha llegado hasta nosotros.

\footnotetext{
1 Plin., n.h. 3.24: Caesaraugusta...ubi oppidum antea vocabatur Salduvia, regionis Sedetaniae.

2 Galve IzQuierdo 1995; 2011; FATÁs - Beltrán Lloris 1997.

3 Cf. Beltrán Lloris 2007, 29-30.

4 FERrER 1986.

5 Domínguez 1979; 1997; GARcíA-Bellido - BlázQuez 2001, 71-72.
} 
Ninguno de los dos parece conocer Salduie o, cuando menos, no le conceden -ellos o sus fuentes de información-la suficiente importancia como para hacer mención de ella.

Para el período de la guerra sertoriana, desarrollada en la década de los años setenta del siglo I a.C. y que tuvo una repercusión muy especial en la parte central del valle del Ebro, contamos con fragmentos de las Historiae de Salustio, con la biografía que de Sertorio escribió Plutarco, y con un extenso fragmento del libro 91 de Livio, que se ocupa de las actividades militares y organizativas de Sertorio en la zona en los años 77 y 76 a.C. En el detallado fragmento de Livio se mencionan diversas ciudades próximas al río Ebro: Castra Aelia, ciudad junto a la cual pasa el invierno Sertorio con sus tropas, Contrebia Leucade, Bursao, Cascantum, Grachurris, Calagurris, Vareia. ${ }^{6}$ La mayoría han sido localizadas arqueológicamente o son en cualquier caso fácilmente reconocibles por su topónimo, semejante al actual, en Borja (Bursao), Cascante (Cascantum), Alfaro (Gracchurris), etc. Plutarco, por su parte, atribuye un papel especialmente relevante en el conflicto a Osca, la actual Huesca, a la que califica como "gran ciudad", y que habría de convertirse en la auténtica capital sertoriana. Apiano y Estrabón sitúan los últimos focos de resistencia sertoriana frente al avance de Cneo Pompeyo Magno en Calagurris, Osca e Ilerda, es decir, en las actuales Calahorra, Huesca y Lérida. De nuevo, no hay ningún rastro de Salduie en los relatos más o menos pormenorizados de estos autores. Por consiguiente, de acuerdo con las fuentes literarias la conclusión más plausible es que Salduie no parece haber desempeñado ningún protagonismo en la guerra sertoriana, durante la cual, de acuerdo con los datos arqueológicos disponibles, muchos poblados del valle del Ebro fueron destruidos y abandonados, temporal o permanentemente. ${ }^{7}$

En definitiva, resulta muy llamativo que las fuentes literarias ignoren por completo la existencia de Salduie-Salduvia. Si bien éste no es un dato absolutamente definitivo, puesto que esas fuentes se conservan de manera fragmentaria, resulta muy significativo si tenemos en cuenta que el valle medio del Ebro es una región que adquirió un largo protagonismo durante el proceso de conquista por parte del Estado romano, tanto cuando los pueblos de cultura ibérica fueron sometidos en los últimos años del siglo III y al comienzo del siglo II a.C., como durante la interminable lucha librada contra los celtíberos a lo largo de todo el siglo II y aun en los primeros decenios del siglo I a.C. Salduie estaba ubicada justo en la frontera entre los tres grandes ámbitos culturales reconocibles en la Península Ibérica en época antigua, el ibérico, el indoeuropeo y el

6 Liv., frag. 1.91: Cum magna iactura militum quattuor et quadraginta diebus Contrebia expugnata relictoque ibi L. Insteio (...) ipse ad Hiberum flumen copias reduxit. Ibi hibernaculis secundum oppidum quod Castra Aelia vocatur, aedificatis ipse in castris manebat, interdiu conventum sociarum civitatium in oppido agebat (...) Ipse cum suo exercitu in Berones et Autricones progredi statuit, a quibus saepe per hiemen, cum ab se oppugnarentur Celtiberiae urbes, inploratam esse opem Pompei conpererat missosque, qui itinera exercitui Romano monstrarent, et ab ipsorum equitibus vexatos saepe milites suos, quocumque a castris per oppugnationem Contrebiae pabulandi aut frumentandi causa accessissent (...) Haec secum agitans Sertorius praeter Hiberum amnem per pacatos agros quietum exercitum sine ullius noxa duxit. Profectus inde in Bursaonum et Cascantinorum et Graccuritanorum fines, evastatis omnibus proculcatisque segetibus ad Calagurrim Nasicam, sociorum urbem venit transgressusque amnem propinquum urbi ponte facto castra posuit... Dimissis eis ipse profectus per Vasconum agrum ducto exercitu in confinio Beronum posuit castra (...) Postero die (...) ad Vareiam, validissimam regionis eius urbem, venit. Cf. Pina Polo - Pérez Casas, 1998; Pina Polo 2000, 35-36.

7 BeLtrán Lloris 2002. 
vascónico-pirenaico ${ }^{8}$ Pero, además, en el punto que constituyó durante decenios a lo largo del siglo II a.C. el límite del territorio bajo control de Roma en el avance de las legiones romanas de este a oeste. En ese escenario, sería de esperar que Salduie hubiera desempeñado algún papel protagonista o que, cuando menos, fuera mencionada en alguna ocasión, pero no hay ni rastro de ella.

Las fuentes no hablan de ella en el momento inicial de la conquista, cuando los romanos se apoderaron del territorio ibérico costero y llegaron hasta la región central del valle del Ebro, en el que el llamado ager Sedetanorum, es decir, el territorio de los sedetanos a los que pertenecía Salduie, fue uno de los escenarios en los que los ejércitos romanos hubieron de enfrentarse a las últimas rebeliones indígenas en la zona. ${ }^{9}$ Durante el siglo II, e incluso en el siglo I a.C., Roma se enfrentó repetidamente a los pugnaces celtíberos situados en el área del Jalón y del Moncayo. Salduie-Salduvia estaba situada en una posición estratégica a las puertas del territorio celtibérico. A pesar de ello, ninguno de los historiadores que relatan la conquista la mencionan en ningún momento, algo que llama la atención si tenemos en cuenta que esos mismos historiadores conocen y citan en relación con la conquista romana un buen número de ciudades en el valle del Ebro y decenas de ellas en el conjunto de Hispania, algunas supuestamente de menor importancia y muchas de ellas de ubicación desconocida.

Sin embargo, los datos epigráficos parecen apuntar una visión diferente. En el denominado Bronce de Áscoli se recoge la concesión de la ciudadanía romana -y de otras recompensas menores- a los miembros de un escuadrón de caballería formado por jinetes hispanos. ${ }^{10}$ Quien concedió la ciudadanía fue Cneo Pompeyo Estrabón, padre del más tarde famoso Pompeyo Magno y comandante en jefe de las tropas romanas concentradas en el frente centro-septentrional de Italia durante la guerra de los aliados, conflicto que enfrentó entre los años 91 y 88 a.C. a Roma con buena parte de sus socios itálicos, empeñados en lograr su plena inclusión jurídica en el Estado romano. La decisión de conceder tales premios fue tomada con el consentimiento del consejo (consilium) que asesoraba al general (llamado imperator en la inscripción), cuyos miembros aparecen reseñados en el Bronce. El acto oficial de entrega de las diversas condecoraciones tuvo lugar en el campamento militar ubicado frente a la ciudad de Asculum (la actual Áscoli), en la región italiana del Piceno, el día 17 de noviembre del año 89 a.C. (más probablemente que el año 90, también posible teóricamente, puesto que el asedio de Asculum se prolongó durante dos años consecutivos).

En el documento epigráfico, los jinetes hispanos reciben genéricamente el nombre de turma Salluitana, es decir, el "escuadrón salluitano". ${ }^{11}$ Olvidada ya la vieja tesis de que esta denominación pudiera derivar del no atestiguado nombre del jefe del escuadrón (supuestamente llamado Salluito o Salluitor), existe hoy en día acuerdo en que el adjetivo Salluitana procede de la ciudad denominada, según hemos visto, Salduie en las monedas y Salduvia en su versión latinizada. El hecho de que la turma tomara su nombre de Salduie-Salduvia y no de alguna de las demás ciudades mencionadas en

\footnotetext{
8 FATÁs 1998.

9 Liv., 28.24; 28.31.

10 CIL I², 709; ILS 8888. CRiniti 1970; Roldán 1986; Marco Simón 1988.

11 LÁzARo 1986.
} 
el epígrafe es explicado partiendo de la base de que en ella se habría llevado a cabo el reclutamiento de todos los jinetes antes de partir hacia Italia.

Casi exactamente al mismo momento corresponde el llamado Bronce de Contrebia. ${ }^{12}$ En esta tabula se da noticia de un pleito surgido en el año 87 a.C. entre los Salluienses, es decir, los habitantes de Salduie, y los Allavonenses, habitantes de la ciudad denominada Alaun según sus monedas, Allavona según las fuentes literarias, habitualmente identificada como la actual Alagón, localidad situada a 25 kilómetros de Zaragoza siguiendo el Ebro aguas arriba en su margen derecha.

Los de Salduie pretendían construir una canalización de agua, muy probablemente para usos agrícolas, en un terreno que habían adquirido a los sosinestanos, miembros de una comunidad de la que desconocemos su ubicación, pero que debía de estar situada al sur del Ebro, entre Salduie y Alaun. Los de Alaun impugnaban la venta, puesto que reclamaban la propiedad de los terrenos en cuestión, o al menos ciertos derechos sobre ellos. Hay que suponer que, ante la imposibilidad de llegar a un acuerdo entre ambas comunidades, se decidió acudir al arbitraje del consejo de notables o "senado", según la denominación que se le adjudica en la inscripción, de una tercera ciudad, Contrebia Belaisca, es decir, la actual Botorrita, ubicada a unos 20 kilómetros al sur de Zaragoza. Se da la interesante circunstancia de que las tres comunidades involucradas en el proceso, situadas en un radio de menos de 30 kilómetros de distancia entre sí, pertenecían a los tres ámbitos culturales paleohispánicos conocidos, siendo Salduie una ciudad de lengua ibérica, Contrebia Belaisca de lengua celtibérica, y Alaun una población vascónica de acuerdo con la afirmación del geógrafo griego Ptolomeo, si bien su nombre tiene claras resonancias célticas. El "senado" contrebiense sentenció finalmente a favor de Salduie, y el fallo fue sancionado por el gobernador provincial C. Valerio Flaco, en tanto que máxima autoridad política y judicial del Estado romano en la Hispania Citerior. Tanto las circunstancias del pleito como su resolución fueron redactadas en latín, utilizado como lingua franca entre hablantes de diversos idiomas, y todo ello inscrito sobre bronce para su exposición pública, siguiendo la costumbre romana. ${ }^{13}$ Eso sucedió, según se especifica en la inscripción, el día 15 de mayo del año 87 a.C.

A partir de los datos hasta aquí expuestos, procedentes de las fuentes literarias, numismáticas y epigráficas, existen indicios que hacen pensar que Salduie fue hasta el comienzo del siglo I a.C. una población de importancia secundaria en el valle medio del Ebro o que, tal vez, ni siquiera existió como tal ciudad hasta entonces. Las favorables condiciones naturales del lugar en el que se ubicó la población permiten suponer razonablemente que pudo existir durante largo tiempo en lo que fue su solar un núcleo habitado próximo al Ebro, pero otra cosa distinta es dar por hecho que ese núcleo fue siempre lo que podríamos denominar una ciudad y que ésta se llamara Salduie. Tal vez haya que suponer mejor que, quizás hasta el último tercio del siglo II a.C., lo que existió en esta zona fue un poblamiento disperso, estructurado en torno a pequeños núcleos habitados, siendo uno de ellos el germen de la ciudad llamada Salduie. ${ }^{14}$

12 CIL I², 3951a. FATÁs 1980; RiCHARDSON 1983.

13 Cf. Beltrán Lloris 1995; 1997; 1999.

14 Pina Polo 2005. 
La datación de las monedas salluitanas en la parte final del siglo II y en el primer tercio del I a.C. indican un hecho de gran importancia. La simple circunstancia de que Salduie fuera entonces una ceca en la que se acuñaba moneda con unos tipos iconográficos determinados y con una leyenda que reflejaba su nombre indígena, supone un salto cualitativo que nos indica sin lugar a dudas que entonces sí existía una ciudad con esa denominación. Lo que no nos permite determinar la información a nuestro alcance es si se trataba de un centro urbano de nueva fundación o de una ciudad resultante del crecimiento experimentado por un núcleo preexistente a través quizá de un proceso de sinecismo, con la llegada de habitantes procedentes del entorno.

En el último cuarto del siglo II a.C. encontramos a través de la epigrafía los primeros datos seguros que evidencian el inicio por parte del Estado romano de la construcción de una red viaria en la región, articulada en torno a dos grandes vías, una paralela a la costa mediterránea, la otra paralela al Ebro, al norte del río, que comenzó por unir Tarraco en la costa con Ilerda y Osca en el interior, y que acabó todavía en época republicana por llegar a Pompelo (Pamplona) y Oiasso en el Cantábrico. Junto con esas dos grandes vías, fueron por supuesto construidas o mejoradas otras menores. ${ }^{15}$

Por otra parte, en las décadas en torno al año 100 a.C. parece haberse producido la fundación de ciudades que parecen haber mantenido sus antiguos nombres indígenas, pero trasladando su ubicación unos pocos kilómetros. ${ }^{16} \mathrm{En}$ el nordeste peninsular, sería el caso de Baetulo (Badalona), Iluro (Mataró), Gerunda (Gerona), Iesso (Guissona), Aeso (Isona), Ilerda (Lérida) y tal vez otras. En ese mismo contexto habría que entender ahora la creación de la ciudad romana de Emporion (Ampurias) junto a la colonia griega del mismo nombre. ¿Debemos plantearnos como posible la creación de una ciudad de nueva planta de nombre Salduie en los años finales del siglo II a.C.? Este hecho permitiría comprender el inicio de la actividad de la ceca de Salduie en ese mismo momento, aunque su actividad fuera reducida, así como la posterior aparición de la ciudad en los bronces de Ascoli y Contrebia.

Tras la supuestamente definitiva conquista de los celtíberos una vez vencida Numancia, en el final del siglo II y comienzo del I a.C. se vivió una nueva guerra celtibérica, como lo muestran los triunfos y ovaciones celebradas en Roma por varios generales. ${ }^{17}$ Según Apiano, los celtíberos fueron derrotados por Cayo Valerio Flaco, que celebraría a su regreso a Roma en el año 82 a.C. un solemne triunfo por su victoria. En el enfrentamiento murieron de acuerdo con Apiano 20.000 celtíberos. ${ }^{18}$ En esos años, Salduie volvió a estar en una zona fronteriza, relativamente próxima al escenario bélico. Pero fue precisamente a partir del momento en que los celtíberos volvieron a ser pacificados, y coincidiendo con la estancia de Valerio Flaco, cuando Salduie empezó a adquirir relevancia. Tal vez no sea una causalidad que los salluitanos cobraran protagonismo en los dos documentos epigráficos mencionados, el Bronce de Áscoli y la Tabula Con-

15 Magallón Botaya 1987.

16 Pina Polo 1993. Considero que la hipótesis formulada entonces de un selectivo traslado de ciudades indígenas a otro emplazamiento cercano, en la parte final del siglo II y en el primer tercio del siglo I a.C., sigue siendo válida, aunque obviamente los datos arqueológicos conocidos sobre algunas de las ciudades han variado en los últimos veinte años.

17 Pina Polo 2009, 224-226.

18 App., Iber: 100. 
trebiensis, datados como hemos visto poco después del final de las hostilidades contra los celtíberos, respectivamente en los años 89 y 87 a.C. Quizá nos encontremos en una fase clave en la historia de la ciudad, un tiempo de consolidación y auge. En esa fase de crecimiento podría entenderse la querella con los vecinos allavonenses por la construcción de un canal como un signo de ese desarrollo. El hecho de que se concediera a los salluienses el derecho a construir el canal, derecho sancionado por el gobernador romano, que no es otro que el propio Valerio Flaco, quizá pueda verse en un contexto de favor por parte del Estado romano hacia la pujante comunidad del Ebro. Con todo, hay que insistir en que el hecho de que Salduie no sea mencionada durante la guerra sertoriana plantea dudas sobre el nivel real de crecimiento de la ciudad.

Por otro lado, si es cierta la hipótesis según la cual la turma Salluitana fue llamada así porque Salduie-Salduvia fue un centro de reclutamiento en el valle del Ebro durante, al menos, el Bellum Sociale, ¿cuál fue la razón de esa distinción? Teóricamente, una posible respuesta podría encontrarse en un mayor peso numérico dentro del escuadrón de los combatientes procedentes de Salduie: habría sido supuestamente práctico concentrar a todos los jinetes en la ciudad de donde provendrían la mayoría de ellos. Sin embargo, los Salluitani que formaban parte de la turma eran tan sólo cuatro del total de treinta componentes, siendo la opinión comúnmente aceptada que Salluitani eran los cuatro citados en primer lugar, cuyo nombre no va precedido de la mención de su ciudad de origen, como sucede con todos los demás. En realidad, los Salluitani no sólo no componían la mayoría del escuadrón en términos absolutos, sino que ni siquiera eran el contingente más numeroso en términos relativos, puesto que se veía ampliamente superado por los segienses, que aportaban nueve, e igualado por los procedentes de una ciudad desconocida para nosotros, cuyos habitantes son llamados en el epígrafe [...]licenses.

Se ha apuntado habitualmente como explicación un supuesto carácter de capitalidad política y administrativa de Salduie en el valle del Ebro, prefigurando el papel asumido decenios más tarde por Caesar Augusta. En mi opinión, este argumento no es válido por tratarse de una anticipación histórica, puesto que la importancia alcanzada posteriormente por Caesar Augusta en ningún caso puede hacerse extensiva a la anterior Salduie. De acuerdo con los datos que han sido expuestos con anterioridad, difícilmente se puede atribuir a la ciudad de Salduie una preeminencia en el conjunto del valle del Ebro que justifique su designación como centro de reclutamiento de la turma Salluitana. En esa época, otras ciudades del área gozaban evidentemente de mayor importancia, y entre ellas se encontraba sin duda Ilerda (Lérida), que aportaba tres jinetes al escuadrón, excepcionalmente con nombres ya latinizados, y que hubiera podido con más razón ser el punto de encuentro de los jinetes, si de elegir la población más relevante se hubiera tratado. De hecho, el proceso pudo ser justamente el inverso: la designación -circunstancial o tal vez permanente- de Salduie como centro de reclutamiento, lo que posiblemente presuponía algún tipo de presencia administrativa del Estado romano en la ciudad, unida a sus condiciones geográficas, pudo ser a largo plazo una de las causas de que sobre ella se fundara la gran colonia romana del valle del Ebro.

En mi opinión, las razones que llevaron a quien era entonces el gobernador de Hispania Citerior, Cayo Valerio Flaco, a designar Salduie como centro de reclutamiento, previsiblemente al comienzo del año 90 a.C., fueron estrictamente de orden práctico. 
Teniendo en cuenta las circunstancias que rodearon el comienzo de la guerra de los aliados, para el Estado romano era de importancia capital que los soldados que se incorporaran a su ejército procedentes de las provincias lo hicieran en el plazo más breve de tiempo posible, para cubrir los enormes huecos que la rebelión de los itálicos había dejado entre las tropas auxiliares permanentes. Por esa razón fue elegida Salduie, que unía a su centralidad geográfica en el valle medio del Ebro su ubicación junto al río, lo que facilitaba el rápido traslado de los reclutados a Italia. ${ }^{19}$

Este es un punto de vital importancia para comprender el auge de Salduie-Salduvia a lo largo del siglo I a.C.: la navegabilidad del Ebro. Como sabemos por un texto de la Historia natural de Plinio, ${ }^{20}$ el Ebro era navegable hasta la ciudad de Vareia, es decir, hasta lo que en la actualidad es Logroño. Esto significa que el Ebro era navegable en la Antigüedad a lo largo de unos trescientos cincuenta kilómetros, y que la actual Zaragoza estaba ubicada aproximadamente en un lugar central de ese curso navegable, casi equidistante de Vareia, el punto final máximo de las navegaciones, y de Dertosa, la actual Tortosa. Dertosa era la gran ciudad del bajo Ebro, a cuyo puerto, tanto fluvial como marítimo, parece que podían acceder en época antigua grandes barcos, por lo que, aunque es indudable que la cercana Tarraco (Tarragona) era el gran puerto marítimo de la región desde su creación al final del siglo III a.C., no hay que descartar que también de Dertosa partieran naves en dirección a Italia, al igual que barcos tipo laúd río arriba, que han seguido surcando el bajo Ebro hasta la segunda mitad del siglo XX..$^{21}$ En cualquier caso, es evidente la enorme importancia que el carácter portuario de Dertosa tuvo en el desarrollo de la población, como se ve en los tipos de sus monedas, en las que aparecen representados barcos como símbolo de la ciudad. ${ }^{22}$

Salduie ocupó por lo tanto un lugar estratégico en la cuenca del Ebro: lugar central fácilmente accesible por vía fluvial y por tierra (aunque no parece que tuviera entonces todavía un papel esencial en la red viaria como lo tendría más tarde la colonia Caesar Augusta), estaba además situada en un vado natural del río donde más tarde se construiría un puente permanente. ${ }^{23}$ En ella debió de existir desde el principio un puerto fluvial que llegaría a tener una gran importancia tras la fundación de la colonia. ${ }^{24}$ Valerio Flaco debió de permanecer como gobernador en Hispania Citerior casi una década, en la que pudo conocer bien el territorio. ¿Fue él el impulsor del desarrollo de Salduie, como indicaría su elección como lugar de reclutamiento durante la guerra de los aliados? Sea como fuere, ese desarrollo debió de ser paulatino más que repentino. En ningún caso deberíamos atribuir a Salduie un papel de capitalidad en el valle medio del Ebro que no tenía. De hecho, cuando la guerra civil entre cesarianos y pompeyanos finalizó, tras la muerte de César no fue en Salduie donde el triunviro Lépido fundó la primera colonia

\footnotetext{
19 Pina Polo 2003.

20 Plin., n.h. 3.21.

21 En general sobre la navegación en el Ebro, véase el clásico de CARrera I CANDi 1993. También BELTRÁN MARTínez 1999. En clave de novela, pero con un gran volumen de información práctica, MoNCADA 1989.

22 IzQuierdo i Tugas 1990; Genera i Monells 1995.

23 Sobre las condiciones naturales del solar en el que se asentó Salduie y luego Caesar Augusta, véase Ollero 1996; Peña - Longares - Constante 2013.

24 Aguarod - ERice 2003.
} 
de ciudadanos romanos en el valle del Ebro, sino en Celsa (Velilla de Ebro), en el año 42 a.C. El cambio decisivo y definitivo se produjo realmente cuando César Augusto decidió fundar una colonia con su nombre..$^{25}$ La ibérica Salduie dejó paso a la colonia Caesar Augusta, una ciudad hispano-romana habitada como dice Estrabón por romanos e indígenas. ${ }^{26}$ Desde entonces, Caesar Augusta habría de convertirse en una ciudad mestiza y en el principal centro político y administrativo del valle del Ebro, capital del conventus iuridicus Caesaraugustanus.

\section{Bibliografía}

Aguarod, C. - Erice, R. (2003): "El puerto de Caesaraugusta", [en] Puertos fluviales antiguos: ciudad, desarrollo e infraestructuras. IV Jornadas de Arqueología Subacuática, Valencia, 143-155.

BeLtRÁn LloRis, F.

(1992): "Caesar Augusta, ciudad de Augusto", Caesarangusta 69, 31-44.

(1995): "La escritura en la frontera. Inscripciones y cultura epigráfica en el valle medio del Ebro", [en] F. Beltrán Lloris (ed.), Roma y el nacimiento de la cultura epigráfica en Occidente, Zaragoza, 169-195.

(1997): "Inscripciones sobre bronce: ¿un rasgo característico de la cultura epigráfica de las ciudades hispanas?, [en] XI Congresso Internazionale di Epigrafia Greca e Latina (Roma, 18-24 settembre 1997), Roma, 22-37.

(1999): "Writing, language and society: Iberians, Celts and Romans in Northeastern Spain in the 2nd and 1st centuries BC", BICS 43, 131-151.

(2002): "La etapa de Sertorio en el valle del Ebro. Bases arqueológicas", Pallas 60, 45-92.

(2007): "Topografía y evolución urbana", [en] F. Beltrán Lloris (ed.), Zaragoza. Colonia Caesar Augusta, Roma, 29-42.

Beltrán MartíneZ, A. (1999): "El valle del Ebro en época romana. Noticias de los historiadores y geógrafos", [en] Hiberus flumen. El río y la vida, Zaragoza, 45-62.

CARRERA I CANDI, F. (1993, $\left.{ }^{1} 1940\right)$ : La navegació al riu Ebre, Barcelona.

Criniti, N. (1970): L'epigrafe di Asculum di Gn. Pompeo Strabone, Milano.

Domínguez, A.

(1979): Las cecas ibéricas del Valle Medio del Ebro, Zaragoza.

(1997): "Las acuñaciones ibéricas y celtibéricas de la Hispania Citerior", [en] Historia monetaria de Hispania antigua, Madrid.

FATÁs, G.

(1980): Contrebia Belaisca (Botorrita, Zaragoza) II. Tabula Contrebiensis, Zaragoza. (1998): "El Ebro medio, trifinio paleohispánico", [en] J. F. Neila - F. J. Navarro (eds.), Los pueblos prerromanos del norte de Hispania. Una transición cultural como debate histórico, Pamplona, 29-50.

Fatás, G. - Beltrán Lloris, M. (1997): Historia de Zaragoza 1: Salduie, ciudad ibérica, Zaragoza.

25 Beltrán Lloris 1992.

26 Str., 3.2.15. 
FERrER, Ma J. (1986): “El nombre prerromano de Zaragoza”, Caesaraugusta 63, 17-47.

GALVE IZQUIERDo, $\mathrm{M}^{\mathrm{a}} \mathrm{P}$.

(1995): Los antecedentes de Caesaraugusta. Estructuras domésticas de Salduie, Zaragoza. (2011): "Salduie, ciudad ibérica", [en] I. Aguilera - J. L. Ona (coords.), Delimitación comarcal de Zaragoza, Zaragoza, 86-87.

García-Bellido, Ma P. - Blázquez, C. (2001): Diccionario de Cecas y Pueblos Hispánicos (Vol. I y II), Madrid.

Genera i Monells, M. (1995): "El curso inferior del Ebro: vía de difusión y contactos durante la Romanización”, [en] XXI Congreso Nacional de Arqueología, Zaragoza, 143-154.

IzQUIERDO I Tugas, P. (1990): "Liaisons entre navigation maritime et fluviale en Tarraconaise: les exemples de Les Sorrres et Dertosa”, [en] Le commerce maritime romain en Méditerranée occidentale, PACT 27, 189-199.

LÁzAro, R. (1986): “La ecuación Salluitanus/sallviensis”, [en] Reunión sobre epigrafía hispánica de época romano-republicana, Zaragoza, 137-139.

Magallón Botaya, Mª A. (1987): La red viaria en Aragón, Zaragoza.

Marco Simón, F. (1988): “Soldados del Ebro medio en la Roma antigua”, [en] AA.VV., Aragón en el mundo, Zaragoza, 36-48.

Moncada, J. (1989): Camino de sirga, Barcelona.

Ollero, A. (1996): El curso medio del Ebro: geomorfología fluvial, ecogeografía y riesgos, Zaragoza.

Peña, J. L. - Longares, L. A. - Constante, A. (2013): "El marco geográfico del solar de Cae-

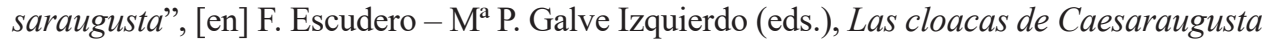
y elementos de urbanismo y topografia de la ciudad antigua, Zaragoza, 33-47.

Pina Polo, F.

(1993): “¿Existió una política romana de urbanización en el Nordeste de la Península Ibérica?", Habis 24, 77-94.

(2000): “La conquista”, [en] F. Beltrán - M. Martín-Bueno - F. Pina Polo (eds.), Roma en la cuenca media del Ebro. La Romanización en Aragón, Zaragoza, 15-45.

(2003): “¿Por qué fue reclutada la Turma Salluitana en Salduie?”, Gerión 21, 197-204.

(2005): "De Salduie a Caesaraugusta: La creación de una ciudad hispano-romana", [en] J. J. Domingo Frax (coord.), Zaragoza, espacio histórico, Zaragoza, 5-15.

(2009): "Hispania y su conquista en los avatares de la República tardía", [en] J. Andreu - J. Cabrero - I. Rodà (eds.), Hispaniae. Las provincias hispanas en el mundo romano, Tarragona, 223-236.

Pina Polo, F. - Pérez Casas, J. Á. (1998): "El oppidum Castra Aelia y las campañas de Sertorius en los años 77-76 a.C.”, Journal of Roman Archaeology 11, 245-264.

Richardson, J. S. (1983), "The Tabula Contrebiensis: Roman law in Spain in the early first century", JRS 73, 33-41.

Roldán, J. M. (1986): "El bronce de Ascoli en su contexto histórico", [en] Reunión sobre epigrafía hispánica de época romano-republicana, Zaragoza, 115-135 (=Ejército y sociedad en la España romana, Granada, 1989, 119-148). 